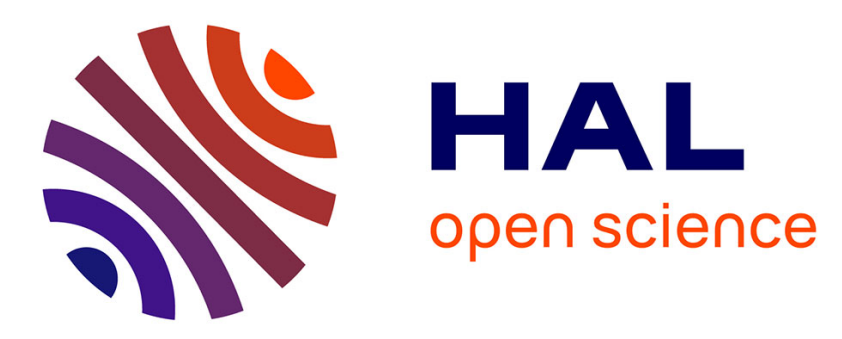

\title{
Individualisation de parcours d'apprentissage : potentiel de blogs
}

Catherine Loisy

\section{To cite this version:}

Catherine Loisy. Individualisation de parcours d'apprentissage: potentiel de blogs. STICEF (Sciences et Technologies de l'Information et de la Communication pour l'Éducation et la Formation), 2012, Individualisation, personnalisation et adaptation des Environnements Numériques d'Apprentissage, 19, pp.253-283. 10.3406/stice.2012.1047 . hal-00842924

\section{HAL Id: hal-00842924 \\ https://hal.science/hal-00842924}

Submitted on 12 Apr 2016

HAL is a multi-disciplinary open access archive for the deposit and dissemination of scientific research documents, whether they are published or not. The documents may come from teaching and research institutions in France or abroad, or from public or private research centers.
L'archive ouverte pluridisciplinaire HAL, est destinée au dépôt et à la diffusion de documents scientifiques de niveau recherche, publiés ou non, émanant des établissements d'enseignement et de recherche français ou étrangers, des laboratoires publics ou privés. 


\title{
Individualisation de parcours d'apprentissage : potentiel de blogs
}

\author{
Catherine LOISY (IFE - ENS-Lyon)
}

- RÉSUMÉ : Cet article examine l'individualisation de parcours d'apprentissage, dans le cadre de l'utilisation de blogs. La théorie des genèses instrumentales permet de définir les caractéristiques de l'environnement numérique soutenant la construction de compétences relatives à l'identité numérique et à l'orientation; elle conduit à mettre en place des e-portfolios. La notion de tutorat donne un cadre pour analyser la gestion de l'apprendre. La recherche interroge les pratiques d'enseignants de trois classes du secondaire. La manière dont les enseignants gèrent les apprentissages de leurs élèves est analysée en termes d'acceptabilité des artefacts, et de soutien des dimensions cognitives et affectives dans l'apprentissage. Par rapport à l'individualisation des apprentissages, des variations sont observées entre les données collectées, mais elles ne sont pas liées à des différences de compétences numériques des enseignants.

- MOTS CLÉS : Individualisation, e-portfolio, identité numérique, orientation active, genèses instrumentales, gestion de l'apprendre.

ABSTRACT : The present paper examines individualization of learning paths, in the context of the use of blogs. The theory of instrumental genesis permits defining the characteristics of the digital environment supporting development of competencies related to digital identity and guidance, and leads to the implementation of e-portfolios. Tutoring provides a framework for analyzing learning management. This research examines the practices of teachers from three high school classes. Teacher management of student learning is analyzed in terms of the acceptability of artefacts, and of supporting cognitive and affective dimensions in learning. In relation to the consideration of individualized learning, there are differences between the collected data, but these cannot be explained by differences in the teachers' digital competencies.

KEYWORDS : Individualization, e-portfolio, digital identity, career guidance, instrumental genesis, learning management.

- 1 . Introduction

- 2. Cadre théorique

- 3. Méthodologie

- 4 . Résultats

- $\underline{\text { 5. Intérêt des blogs dans l'individualisation de parcours d'apprentissage }}$

- REMERCIEMENTS

- BIBLIOGRAPHIE

\section{Introduction}

En France, les compétences relatives à l'informatique et l'Internet des élèves sont désormais évaluées à tous les niveaux de la scolarité obligatoire. Cependant, avec l'évolution constante des potentialités du numérique, de nouvelles questions apparaissent régulièrement qui soulignent les limites des référentiels. Ainsi, avec l'essor des réseaux sociaux, se pose la question de l'identité numérique. Parallèlement, pendant longtemps, l'orientation des élèves en France était décidée par l'institution, mais les choses changent ; aujourd'hui, il est demandé à l'élève de s'investir dans son projet d'orientation et les compétences relatives à ce projet sont évaluées officiellement en fin de collège. La construction du projet d'orientation passe par une réflexion sur soi : il faut permettre à la personne à la fois de conscientiser les 
formes identitaires qui lui paraissent souhaitables et d'identifier les activités et interactions susceptibles de permettre de se construire dans ces formes (Guichard et Huteau, 2005). Pour ces deux visées, l'identité numérique et l'orientation, la construction de l'identité est centrale. Dans ce contexte, le projet $\mathrm{INO}^{(1)}$ (Identité numérique et orientation) vise à faire en sorte que les enseignants construisent des situations pédagogiques dans lesquelles les élèves travaillent leur projet d'orientation tout en développant des compétences relatives à leur identité numérique. Soutenir cette construction identitaire potentiellement permise nécessite une individualisation des parcours d'apprentissage et de développement des élèves. Cet article interroge comment cette individualisation des parcours peut exister, via l'usage d'environnements numériques, dans des classes ordinaires, l'une de collège, les autres de lycée.

On parle d'identité numérique pour désigner les représentations d'une personne qui sont présentes dans les systèmes d'informations. L'identité numérique est liée à l'avènement du Web qui offre des possibilités d'expression avec les blogs, les forums, les réseaux sociaux, etc. : on peut observer la multiplication de données personnelles sur la toile. Cette représentation de soi émanant directement de ce que l'usager décide de montrer de lui-même en le saisissant sur la toile est l'identité déclarative au sens de George (George. 2009). A son propos, Cardon (Cardon. 2008) note que les usagers n'hésitent pas à entrer en relation avec des inconnus, voire à montrer des traits de leur identité sur lesquels on aurait pu supposer une certaine réserve. Une étude (Varga et Caron, 2009) indique que, chez les étudiants, une certaine porosité existe entre les sphères privée et institutionnelle "dans la mesure où leurs réseaux sociaux et les amitiés sont souvent liés à leur formation" (p. 12) et alors même que cette porosité n'est pas présente dans leurs représentations. Par ailleurs, l'identité numérique évolue aussi indépendamment des actions volontaires des personnes (George. 2009). L'auteur distingue deux types de représentations de soi sur le Web qui échappent partiellement à l'usager ; l'identité agissante se compose des signes interprétés par les systèmes d'information à partir des activités de l'usager sur la toile (par exemple, les connexions sur Google permettent au système de repérer à quoi s'intéresse l'usager) ; l'identité calculée est le produit des calculs du système d'information (par exemple, le nombre d'amis sur Facebook). Pour Merzeau (Merzeau, 2010), les usagers devraient avoir une visibilité sur la traçabilité et apprendre à anticiper l'indexation que peut mettre en place le système dès le dépôt de leurs données. L'auteur souligne également que, pour contrebalancer cette part passive grandissante, il faut encourager les usagers à participer activement à la production de leur identité et renforcer leur projet d'habiter l'espace numérique.

Pour ce qui concerne l'orientation, le Conseil Européen de Lisbonne (2000) a défini une stratégie pour dix années, le programme Éducation et formation 2010, avec un engagement de 1'Union Européenne à réformer sa politique d'éducation et de formation en donnant une place centrale à l'orientation. La conférence de Jyväskylä (Finlande) en 2006 prône le déploiement de politiques nationales d'orientation tout au long de la vie. En France, depuis 2009, la publication d'une circulaire ${ }^{(2)}$ met l'accent sur la prise en charge, par l'élève, de son orientation. Le texte dit que l'élève doit construire un projet d'orientation en s'appuyant sur sa personnalité, par une prise de conscience de ses aspirations et compétences. On doit également lui donner accès aux possibilités d'orientation scolaire et de métiers. L'élève est accompagné par l'équipe éducative, dont les enseignants. Au collège, le socle commun de connaissances et de compétences $^{(3)}$ (socle commun) précise que l'élève doit développer sa "capacité à s'orienter tout au long de la vie". L'éducation à l'orientation se fonde sur la perception de l'environnement comme mouvant (Pelletier et Dumora, 1984). Dans ce contexte, il s'agit de faire acquérir à l'élève une compétence susceptible de le rendre capable d'analyser les éléments de soi et la structure du monde environnant afin de développer des stratégies d'ajustement, compétences ne s'inscrivant pas uniquement dans le temps présent, mais censées être pérennes.

Comme cela a été mentionné, la construction de l'identité est centrale dans les deux visées du projet, l'identité numérique et l'orientation. Processus psychique majeur, l'identité est un ensemble de "caractéristiques physiques, psychologiques, morales, juridiques, sociales et culturelles à partir desquelles la personne peut se définir, se présenter, se connaître et se faire connaître, ou à partir desquelles autrui peut la définir, la situer, ou la reconnaître" (Tap, 1979, p.8). Sa construction progressive commence dès l'enfance et se poursuit à l'âge adulte dans les confrontations avec d'autres personnes. Pour Jorro (Jorro, 2009, p. 4), "L'engagement dans une dynamique de professionnalisation s'accompagne de prises de consciences successives, parfois coûteuses : nécessité de se connaître pour élaborer un 
projet, de se dégager des formulations vagues pour cibler des enjeux d'apprentissage." Ce cadre parait transposable malgré les différences entre les populations concernées par ces recherches.

Le cadre théorique conduit à proposer l'usage de portfolio numérique ou e-portfolio pour soutenir l'individualisation des apprentissages et du développement et l'articule avec la nécessité de mettre en place une gestion des dispositions à apprendre.

\section{Cadre théorique}

\subsection{Cadre général}

La recherche INO est axée sur une épistémologie ancrée dans le socioconstructiviste où la connaissance se construit dans l'interaction entre un sujet et l'objet à connaître, par le biais d'interactions sociales. Dans la perspective piagétienne e.g. (Piaget, 1937), l'interaction sujet-objet construit à la fois la connaissance de l'objet et participe du mode d'élaboration de la connaissance au sens plus général d'intelligence ; c'est en expérimentant le réel que le sujet construit, et la connaissance de l'objet, et la connaissance de son expérience. Il existe un double mouvement de transformation du milieu par l'action du sujet et de transformation du sujet par son action sur le milieu. Vygotski qui s'intéresse également au développement du sujet, insiste sur le statut social du fonctionnement humain et modélise le rôle d'autrui dans la construction des processus cognitifs, sans ôter le caractère individuel de l'apprentissage. C'est l'interaction sujet-objet-autrui qui construit à la fois la connaissance de l'objet et participe du mode d'élaboration de la connaissance au sens plus général d'intelligence, et qui sous-tend le développement du sujet. Dans cette théorie, l'apprentissage permet le développement.

L'activité humaine est une activité de transformation d'objets. Capable d'être l'acteur de ses dynamiques d'évolution, "à la fois sujet d'activités productives au quotidien et sujet d'activités constructives, par lesquelles il modèle ses systèmes de ressources et de valeurs, ses domaines, situations et conditions d'activités pour le futur" (Rabardel, 2005/2009, p. 13), le sujet produit, c'est-à-dire qu'il agit et transforme des objets du monde qui l'entoure ; il construit, c'est-à-dire que, lorsqu'il produit, son activité le façonne en retour. On parle d'activité constructive pour désigner l'activité qui vise à produire des moyens pour une activité future (Rabardel et Samurçay, 2003).

Pour ce faire, le sujet dispose d'instruments psychologiques qui soutiennent ses actions et son activité de transformation (Vygotski, 1934/1985). En observant de très près l'activité et les dires de jeunes enfants aux prises avec un "problème", l'auteur montre le statut d'instrument du langage : le "langage extérieur", que Piaget qualifie d'égocentrique, joue un rôle dans la prise de distance progressive de l'enfant par rapport à son action, puis il s'intériorise précisément quand il est devenu un instrument suffisamment efficient. Les instruments sont ainsi "incorporés au corps agissant" (Rabardel, 2005/2009, p. 15). Dans le monde contemporain, l'homme dispose de nombreux objets anthropotechniques ou artefacts (Rabardel, $\underline{1995)}$ qui augmentent sa capacité d'action, mais ces artefacts ne sont pas a priori des instruments, ils le deviennent lorsqu'ils acquièrent cette double nature d'extension à la fois interne et externe au sujet, entité alliant composante artefactuelle (artefact matériel ou symbolique) et composante schématique (schèmes associés). Les instruments sont avant tout des moyens d'actions ; le sujet développe des instruments pour soutenir son action de transformation des objets de son environnement. Ces instruments médiatisent les rapports du sujet aux objets de son activité ; du sujet à lui-même ; du sujet à autrui.

Quatre types de médiations dans l'activité productive et dans l'activité constructive peuvent être distingués (Béguin et Rabardel, 2000) :

- les médiations pragmatiques orientées vers l'action sur l'objet de l'activité ;

- les médiations épistémiques orientées vers la connaissance de l'objet lui-même ;

- les médiations heuristiques ou réflexives orientées vers la connaissance et la gestion de soi, par exemple, en anticipant comment l'activité actuelle pourra orienter l'activité future ;

- les médiations interpersonnelles orientées vers la gestion de toutes les dimensions dans lesquelles autrui est partie prenante. 
La figure 1 illustre ce modèle de développement du sujet.

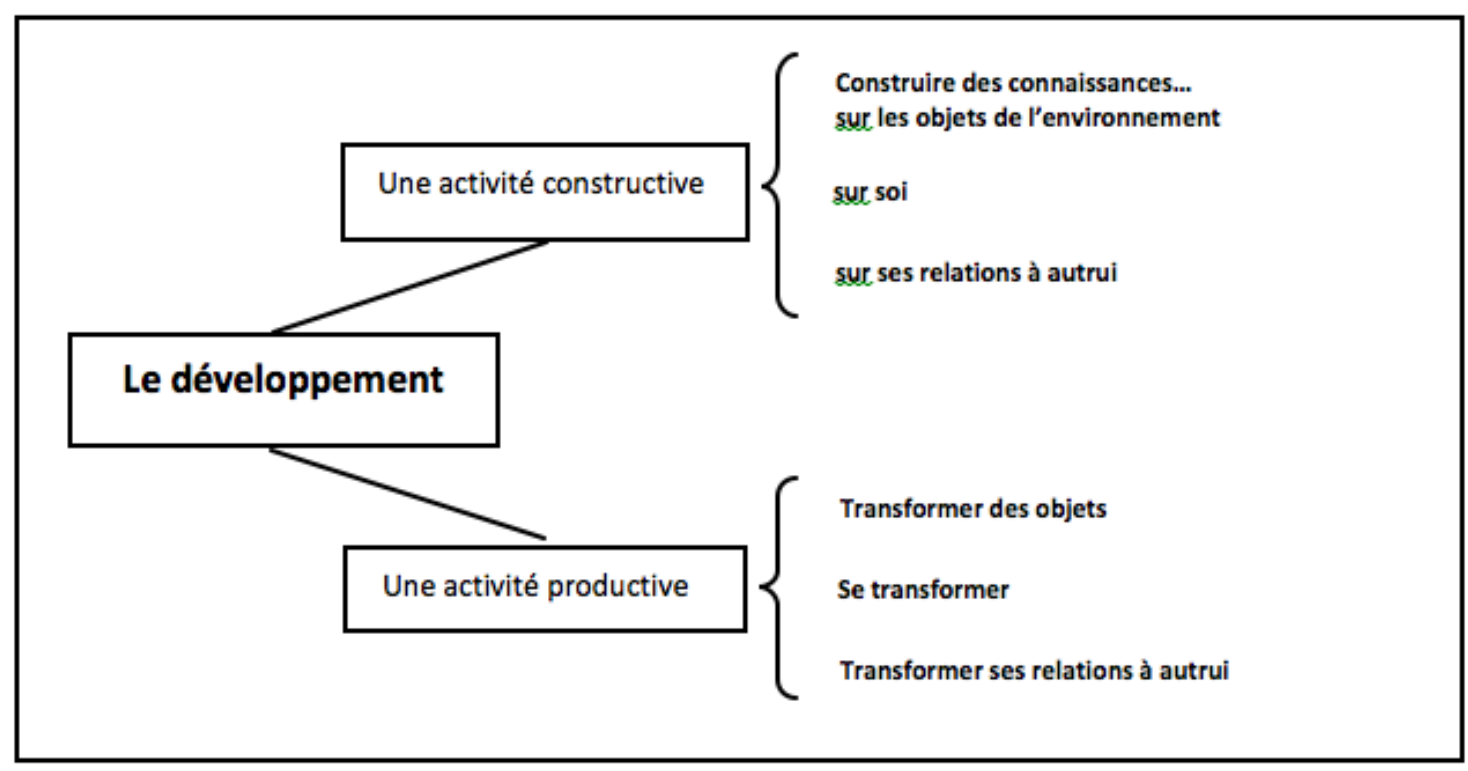

Figure 1 • Le développement du sujet

Ce cadre théorique général conduit aussi à poser la question des environnements technologiques qui sont les plus à-même de soutenir l'apprentissage et le développement individuel.

\subsection{Un environnement numérique pour soutenir des activités constructives}

\subsubsection{Portfolio d'apprentissage et développement}

Le développement s'effectue par une action sur des objets, nécessairement située dans un environnement social, et articulée à une réflexion. Ceci fournit un cadre pour penser les caractéristiques des dispositifs d'apprentissage : un environnement soutenant la genèse d'un instrument qui devrait articuler les trois types de rapports qu'un sujet peut entretenir avec le réel, en l'occurrence le rapport à l'objet de l'activité, le rapport à soi-même, le rapport à autrui. Il s'agit de soutenir la construction de quatre types de médiations mises en évidence par la psychologie du développement : les médiations pragmatiques orientées vers l'action, les médiations épistémiques orientées vers la connaissance des objets, les médiations réflexives orientées vers la connaissance et la gestion de soi ; les médiations interpersonnelles orientées vers la gestion des rapports à autrui.

La figure 2 illustre comment le portfolio d'apprentissage soutient le développement du sujet. 


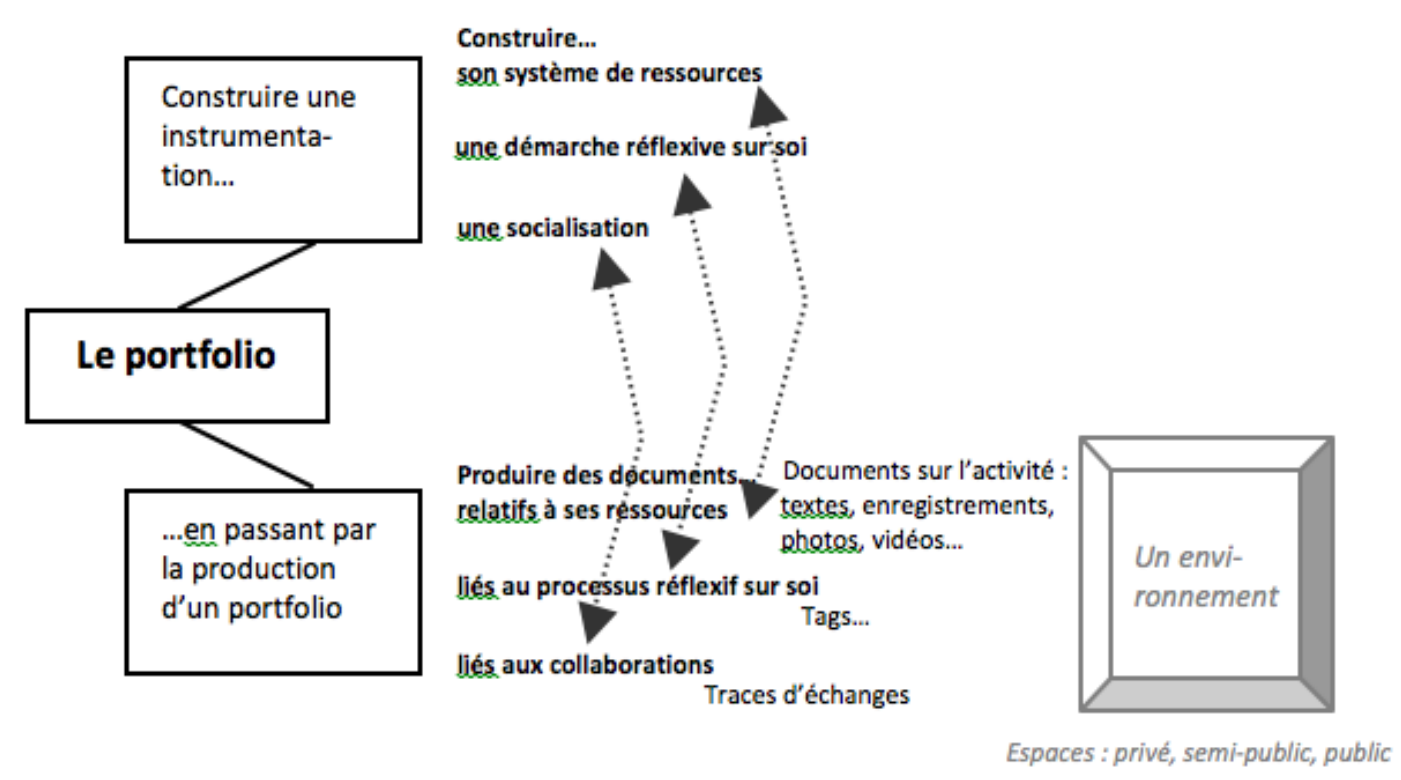

Figure 2 - Portfolio d'apprentissage et développement du sujet

Le portfolio, collection de documents et de réflexions que le sujet récolte et organise, soutient son développement :

- Le portfolio prend forme dans un « objet-portfolio » que le sujet conçoit et développe lui-même ; les dimensions pragmatiques et épistémiques sont entremêlées puisque la production du portfolio et les connaissances sur le portfolio sont étroitement imbriquées. Layec (Layec, 2006) montre comment des étudiants se forment aux méthodologies et outils proposés tout en pilotant leur démarche portfolio ;

- La principale fonction du portfolio est l'analyse réflexive de son auteur sur lui-même et sur ses propres activités (Allal, 1999) ; le portfolio soutient par nature les médiations réflexives. Celles-ci doivent se dérouler, de préférence, dans un espace totalement privé du portfolio ;

- Enfin, le portfolio participe de médiations interpersonnelles : il peut être utilisé dans des espaces plus ou moins ouverts (privé/semi-public/public), permettant de ménager des possibilités d'échanges à différents niveaux, avec différents acteurs; les espaces semi-publics permettent à des personnes choisies d'être partie prenante de l'activité et d'organiser des échanges constructifs.

La version numérique, e-portfolio, offre de nombreux avantages : un espace de stockage important, une grande maniabilité, une facilité d'organisation-réorganisation des données stockées, un transfert aisé d'un espace à un autre, des possibilités d'ouverture quasi infinies. Il permet également de stocker des documents de natures diverses : documents écrits, mais aussi photographies, documents sonores, vidéos... Il offre des possibilités d'annotation, comme les tags. Le e-portfolio ne change pas fondamentalement la démarche mais il convient toutefois de veiller à ce que le sujet ne se centre pas seulement sur la simple présentation de soi en occultant la dimension réflexive (Quéré, 2000) ; (Vermersch, 2000) ; (Loisy et al., 2011).

\subsubsection{Caractéristiques du e-portfolio dans le projet INO}

L'instrument médiateur étant à la fois de nature technique et psychologique (Béguin et Rabardel, 2001), dans le cadre d'INO, l'élève devrait produire son portfolio tout en développant un instrument. Il devrait conserver des documents relatifs à ces ressources dans son portfolio. Au fil de cette production, il analyserait ce qu'il produit, notamment les ressources sur les métiers et carrières, celles qui permettent de construire son projet d'orientation, celles qui concernent les attentes sociétales ; l'instrument permettrait alors une médiation épistémique à son portfolio.

L'élève devrait produire des documents relatifs à lui-même et les conserver dans un espace protégé. Il 
construirait ainsi une démarche réflexive sur lui-même qui participerait de la construction de son identité et alimenterait sa réflexion sur les traces qu'il peut laisser sur le Web, son identité numérique, celle-ci se nourrissant des ressources construites sur les attentes sociétales. Le portfolio et les documents qu'il contient donnent également à l'élève une image de lui-même qu'il peut examiner. L'instrument permettrait ainsi une médiation réflexive.

L'élève devrait vivre des interactions notamment via l'espace semi-public de son portfolio, qui permet des interactions ciblées. Il conserverait des documents relatifs à ses collaborations et interactions sociales. Ces interactions pourraient participer à la socialisation en interrelation avec les ressources qu'il construit sur les attentes sociétales et avec son identité. L'instrument constituerait ainsi un médiateur dans son rapport à autrui.

La figure 3 illustre comment il est attendu que le portfolio numérique fonctionne dans le projet INO.

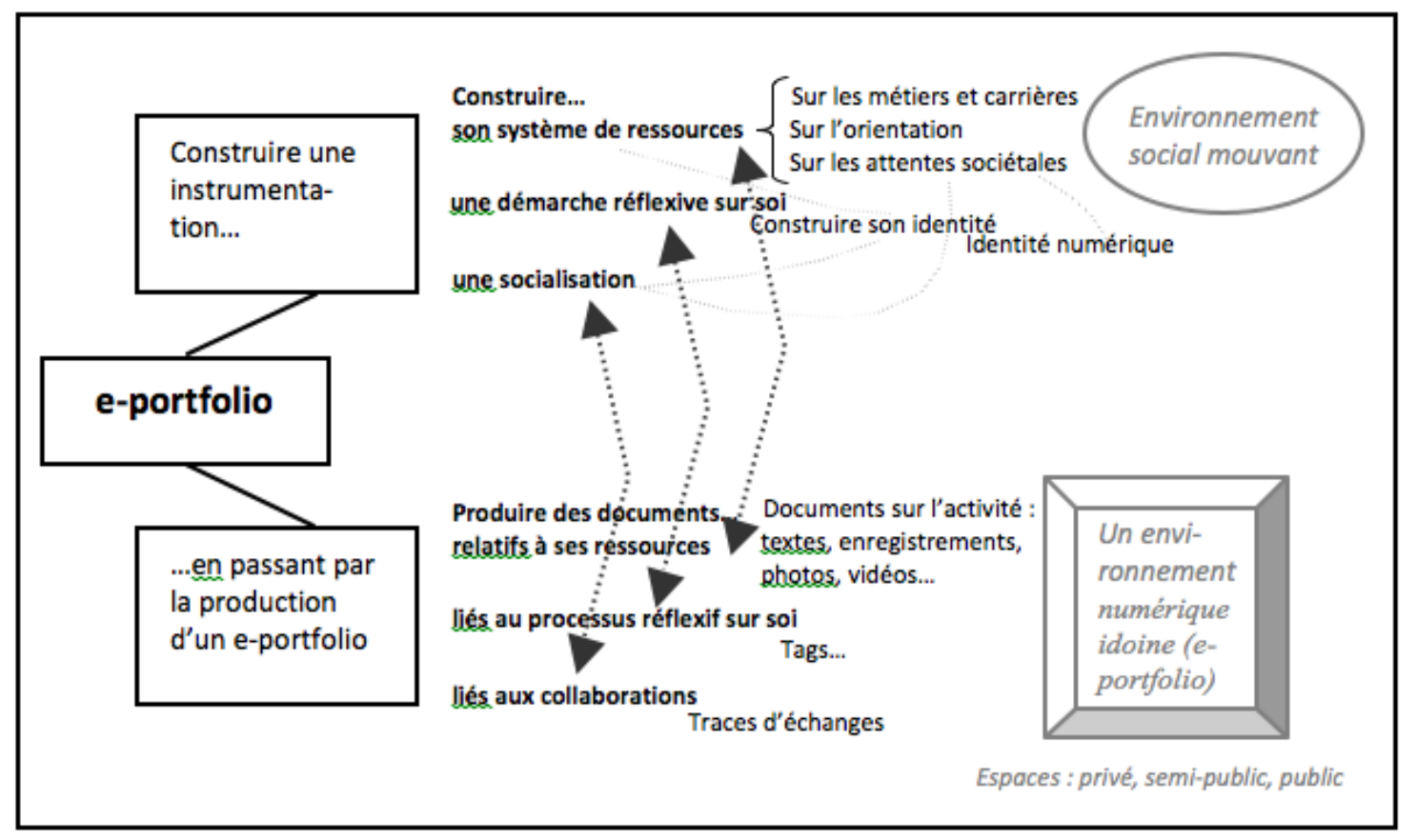

Figure 3 • Le portfolio numérique dans le projet INO

Le modèle illustre l'individualisation des apprentissages avec une visée d'individualisation du développement : il s'agit de faire en sorte que l'élève produise un portfolio avec des documents relatifs à des activités reliées aux trois types de rapport au monde et qu'il construise une instrumentation. Dans la figure 3, les points de centration de cet article correspondent aux flèches avec doubles pointes et aux éléments qu'elles relient.

Cependant, il n'est d'apprentissage que si le sujet lui-même s'engage dans un processus de genèse (Pastré. 2005) : si le portfolio numérique ou e-portfolio est adapté pour les apprentissages et le développement attendus chez les élèves dans le projet INO, l'environnement numérique n'a pas d'effet en lui-même. Apprendre et se développer ne sont possibles qu'avec une activité, un engagement de la part du sujet.

\subsection{La gestion des dispositions à apprendre}

L'intérêt doit se porter sur la gestion des dispositions à apprendre. Cette gestion concerne deux points, la gestion des processus de production avec notamment la prise en compte des caractéristiques des artefacts (dans le but de les rendre acceptables); la gestion des processus de construction avec notamment la prise en compte du coût cognitif et du coût affectif de l'apprendre.

\subsubsection{Des artefacts acceptables}

Les qualités intrinsèques des objets influencent la construction des usages des outils numériques ; 
l'acceptabilité (Tricot et Tricot, 2000) joue un rôle clé puisqu'elle conditionne la décision d'utilisation des environnements numériques. L'acceptabilité renvoie à la représentation mentale de l'utilité (elle-même reliée au but visé par l'artefact) et de l'utilisabilité d'un environnement informatique pour l'apprentissage humain.

Selon la norme ISO 9241-11, l'utilisabilité est "le degré selon lequel un produit peut être utilisé, par des utilisateurs identifiés, pour atteindre des buts définis avec efficacité, efficience et satisfaction dans un contexte d'utilisation spécifié" (source Wikipédia). En d'autres termes, l'utilisateur doit percevoir les artefacts comme permettant d'atteindre les objectifs qu'il vise avec un coût cognitif acceptable, et leur utilisation doit être associée à la satisfaction d'un désir.

Le concept d'utilité peut être rapproché du concept de pertinence (Tricot et Tricot, 2000). Cette dernière est la relation entre deux "groupes" (ibidem, p. 196), celui du document et ses caractéristiques versus celui de la représentation de son problème par l'utilisateur. Cette définition met l'accent sur le rôle de l'utilisateur ; si les artefacts sont conçus pour viser des buts, c'est l'utilisateur qui perçoit ou non leur utilité. Ce couple fonctionne si l'utilisateur a une représentation de son problème et des objectifs qu'il vise en adéquation avec ce que l'artefact peut effectivement l'aider à réaliser.

\subsubsection{Un accompagnement humain}

L'utilité a été définie comme l'adéquation entre ce qu'un artefact peut aider un utilisateur à réaliser et la représentation de son problème. L'utilisabilité a été définie comme la perception que les artefacts permettent d'atteindre les objectifs visés avec un coût acceptable, mais leur utilisation doit aussi être associée à la satisfaction d'un désir. Dans le cadre des activités pédagogiques, c'est le plus souvent l'enseignant qui choisit l'environnement numérique que vont utiliser ses élèves. Une des activités de l'enseignant est alors d'aider les élèves à avoir une représentation du problème en adéquation avec l'utilisation possible de l'artefact, à faire en sorte qu'un désir d'apprendre naisse, à soutenir la motivation à poursuivre.

Pour cela, la notion de tutorat, et plus spécialement le concept d'étayage-désétayage (Bruner, 1983) sont adaptés. L'étayage-désétayage renvoie à un double mouvement ; de soutien pour amener, sans le faire à sa place, un enfant à résoudre un problème qu'il ne sait pas résoudre seul; de retrait progressif lorsque les conduites de résolution du problème de l'enfant se mettent en place. Le terme anglais scaffolding rend bien compte de ce double mouvement de construction de ponts et passerelles soutenus par une charpente, mais qui se caractérise aussi par son caractère temporaire. Bruner associe six fonctions à l'étayage, ces fonctions renvoient aux tâches qui incombent à l'adulte pour soutenir la résolution du problème par l'enfant :

- L'enrôlement est une sorte de motivation initiale, l'adulte amène l'enfant à s'intéresser à l'activité, à se mettre en mouvement ;

- Le maintien de l'orientation concerne le soutien de la motivation de l'enfant à poursuivre la tâche ;

- Le contrôle de la frustration vise à rendre la tâche de l'enfant moins difficile et à l'aider à accepter ses erreurs ;

- La réduction des degrés de liberté par laquelle l'adulte ajuste la tâche aux possibilités de l'enfant en la simplifiant si elle est trop ardue ;

- La signalisation des caractéristiques dominantes qui rend plus saillantes les caractéristiques les plus utiles à la résolution du problème si l'enfant ne les perçoit pas ;

- La présentation de modèle qui n'est pas une démonstration à proprement parler, mais plutôt une proposition de solution s'appuyant sur les réalisations effectives de l'enfant.

Enrôlement, maintien de l'orientation, contrôle de la frustration ont à voir avec le déclenchement de l'activité de l'enfant et le soutien nécessaire au maintien de la poursuite de la tâche. La présentation adoptée ici pour présenter les six fonctions de l'étayage ne correspond pas à leur importance, ni à leur ordre d'apparition, pour soutenir l'apprentissage ; elle vise à rendre saillante l'importance des tâches de l'adulte autour de la mise en mouvement et du maintien à poursuivre qui correspondent à trois sur six des 
fonctions principales de l'étayage. Les tâches de l'adulte sont d'autant plus cruciales que l'enfant est jeune, ainsi que l'auteur (Bruner, 1987) le montre dans son étude sur les interactions mère-enfant dans les processus d'acquisition du langage.

Les travaux de Bruner concernent l'enfant, mais ils peuvent renvoyer à toutes les situations dans lesquelles il y a asymétrie entre les partenaires, de compétences (l'un est expert et l'autre novice), de buts (l'un a pour but de faire agir alors que l'autre a pour but d'agir) ; ce modèle est donc adapté dans le contexte de l'accompagnement du projet INO en classe. Pour aider les élèves à entrer en activité, l'enseignant peut jouer sur le registre affectif, la motivation, et sur le registre cognitif, en ajustant les tâches aux possibilités des élèves, voire en montrant un modèle.

\subsubsection{Caractéristiques de la gestion des dispositions à apprendre dans le projet INO}

Si les qualités du portfolio en font un objet idoine pour apprendre et pour se développer, il n'en demeure pas moins nécessaire que, pour ce faire, l'élève s'engage et entre en activité. Ceci implique que l'enseignant soutienne l'engagement de ses élèves tout au long de l'activité. Il doit les mettre au travail dans un environnement numérique qu'ils perçoivent comme étant utile, utilisable et acceptable, caractéristiques se rapportant à la dimension technique, mais aussi à la dimension cognitive (l'utilité renvoyant à la construction du problème) et affective (l'utilisabilité étant associée au désir d'apprendre). Il doit jouer à la fois sur la motivation et sur le registre cognitif.

L'accompagnement humain vise à soutenir à la fois les processus de production et les processus de construction. Du côté des processus de production, se trouve tout ce qui se rapporte directement à la dimension technique de l'artefact plus tout ce qui vise à simplifier ou rendre accessible l'activité. Du côté des processus de construction, se trouvent les formes d'accompagnement qui visent à soutenir la motivation à entrer en action, le désir d'apprendre et de se développer, le coût cognitif de l'apprendre.

Ce modèle illustre comment l'enseignant peut soutenir l'individualisation des apprentissages et du développement, en greffant un accompagnement humain à un environnement technologique.

\section{Méthodologie}

La recherche, dans son cadre socioconstructiviste, s'appuie sur l'approche collaborative de la recherche en éducation (Desgagné, 1997) où les savoirs se construisent dans une démarche collective d'interprétation qui met en relation théorie et pratiques. Dans cette démarche de co-construction entre membres de l'équipe, si les chercheurs ont initié le projet, en revanche, ils n'ont donné aucune prescription relative aux scénarios pédagogiques, aux ressources à construire, à la mise en œuvre, etc. aux praticiens. Les choses se sont construites chemin faisant et en interaction. Un dispositif d'accompagnement a été mis en place. Ce cadre permet de construire une méthodologie fondée sur un recueil de données "de la pratique", c'est-à-dire d'approcher la pensée active des enseignants, et de savoir comment, confrontés à des situations nouvelles pour eux, ils s'approprient et transforment le réel.

La recherche INO ne répond pas à des attentes des programmes disciplinaires, mais les visées du projet ne sont, en revanche, pas externes aux préoccupations de la sphère éducative. Comme cela a été dit en introduction, la compétence à s'orienter apparaît explicitement dans la compétence 7 du socle commun L'autonomie et l'initiative, au niveau du collège. La compétence $6 \mathrm{du}$ socle commun Compétences sociales et civiques vise également la construction de l'avenir "personnel et professionnel". Si l'identité numérique n'apparaît pas explicitement dans les compétences informatique et Internet définies dans les $\mathrm{B} 2 \mathrm{i}{ }^{\circledR}$ et dans le socle commun, il est attendu que l'élève apprenne à utiliser les technologies numériques "de façon réfléchie" (compétence 4 du socle commun La maîtrise des techniques usuelles de l'information et de la communication).

\subsection{Population et méthodologie générale}

La population est composée, lors de la première année de la recherche, de trois enseignants et d'une conseillère d'orientation psychologue (COP). Dans le lycée, lycée général et technologique important d'une ville moyenne, le projet INO se déroule dans deux classes de seconde, avec un professeur de lettres et un professeur d'anglais. L'établissement accueille des élèves de toutes catégories socioprofessionnelles. 
Les enseignantes considèrent que l'équipement informatique est conséquent. Dans le collège, collège important d'une petite ville, le projet INO se déroule dans une classe de cinquième, avec un professeur de technologie et la COP pour des activités hors les murs de la classe. L'établissement accueille des élèves de classes sociales moyenne et peu favorisée, il y a notamment de nombreux élèves boursiers et une grande partie de la population vit en milieu rural. L'équipe du collège considère que l'équipement informatique est correct.

\subsection{Méthode d'investigation}

Des données sont recueillies de diverses manières et à différents moments. Ici sont précisées les données attendues a priori.

- Données sur l'activité de classe. Ces données sont recueillies tout au long du projet, elles sont déposées librement par les équipes en établissements sur le site collaboratif, le groupe dans son ensemble y a accès. Sont attendus : les produits du processus de conception des situations d'apprentissage, les fiches préparées à l'attention des élèves, des productions des élèves, les liens vers la partie publique du portfolio des élèves, et tout ce qui peut renseigner sur l'activité réelle ;

- Données issues de l'observation de séance. L'équipe de recherche a suggéré de mettre en place des observations croisées de séances dont l'organisation est facilitée par la présence de deux concepteurs dans chaque établissement.

- Données relatives à l'analyse des pratiques professionnelles. Ces données sont recueillies tout au long du projet, notamment sous la forme d'un journal de bord en ligne proposé sous format Excel ;

- Recueil de représentations. Ces données sont recueillies par entretiens à différents moments du projet pour suivre l'avancée du projet dans les classes et sur des thématiques ciblées permettant de faire avancer la recherche. Une première série est prévue à mi-parcours de la première année de la recherche, par équipe d'établissement, au sein même des établissements. La seconde série est prévue en fin de première année de la recherche, en passation individuelle ;

- Bilan du projet. Ces données sont recueillies par un questionnaire auquel il est possible de répondre individuellement ou par équipe à la fin de la première année de la recherche.

\subsection{Méthode d'analyse des données recueillies}

Les journaux de bord en ligne ont été peu renseignés. L'enseignant de collège ne l'a jamais renseigné. Les enseignantes de lycée ont commencé à le renseigner pendant un certain temps au début de la recherche, puis elles ont préféré mettre en place un blog collaboratif relatant leurs réunions de travail internes à l'établissement et leur avancée dans le projet. Il n'y a pas eu non plus d'observations croisées de séances. Les données effectivement recueillies et analysées sont les suivantes :

- Documents élaborés par les praticiens : les documents déposés sur les sites web des praticiens, les diaporamas support des présentations des projets de classes, les journaux de bord ou leurs avatars, des comptes-rendus de réunions, des fiches destinées au travail des élèves, les sites des élèves quand leurs liens ont été transmis ;

- Transcriptions verbatim des entretiens ;

- Réponses au questionnaire final.

Le matériel a été la base d'un travail à visée compréhensive. Nous avons découpé les productions et les discours des répondants en unités informationnelles, puis nous avons classé ces unités par thèmes en fonction des objectifs que nous nous étions fixés a priori tout en restant sensible à l'émergence de nouveaux thèmes. Nous avons ensuite repéré si les unités étaient spécifiques à certains participants ou si elles revenaient dans les productions et discours de tous les participants. Le nombre de participants étant limité, il n'était pas possible d'envisager une analyse statistique.

Ces unités informationnelles se caractérisent par leur sens global unitaire, ce sont le plus souvent des propositions ou des phrases, mais parfois également des groupes de phrases ou simplement des mots pertinents par rapport aux objectifs poursuivis. Les unités linguistiques débordent souvent les unités de 
sens, mais parfois, a contrario, il a été nécessaire de dissocier plusieurs unités de sens à travers une même proposition. Les catégories sont pensées en relation avec les objectifs de l'analyse. Elles sont établies de manière à être exhaustives (toute unité informationnelle doit trouver sa place) et exclusives (chaque unité ne peut appartenir qu'à une catégorie). Pour pallier le risque de biais, plusieurs lectures successives du matériel ont été réalisées.

En relation avec le cadre théorique, l'analyse porte en premier lieu sur les activités constructives en recherchant, dans les productions relatives à la classe, l'existence d'activités d'élèves en lien avec les trois types de rapport au monde : agir pour construire ; réfléchir sur soi ; interagir. Ensuite, elle interroge, à partir des entretiens et des documents relatifs au projet de chaque enseignant, comment sont gérées les dispositions à apprendre, sur le plan des artefacts et sur le plan de l'accompagnement humain.

\section{Résultats}

\subsection{Résultats généraux}

Tous les participants ont déposé des données sur le site web qui leur était dédié. Tous ont accepté de participer aux entretiens qui se sont déroulés comme prévu, la première série en mars, la seconde en juillet, tous ont répondu à toutes les questions qui leur étaient posées. Les entretiens ont été intégralement transcrits. Enfin, le questionnaire final a été renseigné par tout le monde, individuellement au sein de l'équipe de collège, par équipe au sein de l'équipe de lycée.

Le corpus est constitué de diverses données. Leur présentation commence par celles qui sont en lien avec les activités.

- Activités menées en classe sur les thématiques de l'identité numérique et de l'orientation. Un scénario a été recueilli par classe, soit deux scénarios pour le lycée, un scénario pour le collège. De nombreuses ressources afférentes à ces scénarios ont été collectées comme les diaporamas de présentation du projet aux élèves et les fiches préparées à l'attention des élèves, soit pour la construction des compétences, soit pour leur évaluation. Ces documents révèlent que des activités sur l'identité numérique et sur les métiers ont été réalisées dans toutes les classes. Ces données ont été déposées par les enseignants sur le site collaboratif.

- Portfolios numériques. Au lycée, les élèves des deux classes ont créé chacun un blog pour leurs e-portfolios, sauf un élève qui a été très souvent absent et n'a pas été actif dans le projet, aux dire de son enseignante ; ces blogs sont centrés sur la présentation publique de soi. Il n’y a pas eu de portfolios individuels au collège ; lors des entretiens, l'enseignant dit qu'il a fait travailler les élèves en s'appuyant sur les environnements numériques développés par l'ONISEP(4). L'enseignant avait prévu que chacun de ses élèves réalise un diaporama sur un projet de métier, mais cela n'a pas été possible en raison du démarrage du projet INO au second trimestre de l'année scolaire.

Concernant le travail collectif, toute l'équipe "de terrain" était d'accord dès le lancement du projet sur le principe de partager les ressources produites pendant le projet, scénarios et ressources afférentes. Ce sont surtout les enseignants qui ont produit et déposé des ressources sur le site, la COP a toutefois mené des activités avec les élèves de la classe de cinquième du collège sur la connaissance de soi. L'équipe de lycée a donné un accès aux blogs-portfolios des élèves.

Les données de la pratique (documents produits en lien avec la classe), et les extraits des entretiens sont présentés en italiques et entre guillemets dans la suite du texte ${ }^{(5)}$.

\subsection{Le projet INO dans la classe de $5^{\circ}$}

Le binôme du collège, enseignant de technologie et COP, intervient dans la même classe. Au sein de ce binôme, il y a un partage des domaines d'intervention, la partie du projet qui concerne l'orientation est construite et conduite par l'enseignant de technologie, la partie du projet qui concerne l'identité numérique (l'enseignant dit qu'il a parlé de connaissance de soi avec les élèves) est construite principalement par la COP et conduite par elle.

Le projet INO s'intègre au travail réalisé sur la maîtrise des technologies numériques (préparation du 
$\left.\mathrm{B} 2 \mathrm{i}{ }^{\circledR}\right)$ " L'action que nous avons choisie semble bien s'articuler avec les différents aspects traités dans le cours de technologie et devrait en bénéficier sans en modifier le programme" (extrait d'un courriel envoyé par le binôme à l'équipe de recherche à l'issue d'un travail de co-élaboration en début de projet).

Le projet INO est sous-intitulé "Expérimentation du PDMF en classe de cinquième en utilisant le passeport numérique pour l'orientation", ce qui centre d'emblée le projet sur l'utilisation des environnements numériques créés par l'ONISEP. Le projet commence par, et se consacre en grande partie à, des activités sur l'orientation ; cette thématique est abordée par la recherche d'informations dans l'environnement de ressources sur les métiers : "L'activité dominante sera [...] la découverte des métiers". Celles-ci se déroulent dans un environnement numérique.

Au cours de la deuxième réunion de recherche, l'enseignant présente un diaporama sur le Passeport numérique pour l'orientation développé par l'ONISEP. Ce diaporama contient notamment le "programme $d u P D M F$ " pour chaque niveau scolaire. En classe de cinquième, il est stipulé "L'accent est mis sur la découverte des métiers. L'objectif est d'élargir la palette des activités professionnelles appréhendées par les élèves et de développer une méthode d'observation de ces activités." Le document donne des "Exemples de capacités à développer"; il s'agit de décrire le métier d'une personne proche, d'exprimer les qualités requises pour un métier, d'apprendre à utiliser la documentation. La partie du projet qui concerne l'orientation s'appuie sur les objectifs ciblés dans le PDMF. L'activité cible est la "recherche en autonomie articulée avec l'utilisation du Web Classeur".

L'enseignant de technologie et la COP conduisent ensemble les premières activités en classe sur le projet et d'emblée, elles sont centrées sur les environnements numériques développés par l'ONISEP (présentation du projet ; présentation des outils développés par L'ONISEP ; quiz J'explore les métiers). Avec le professeur de technologie, l'élève réalise beaucoup d'activités sur fiche autour des thématiques de la découverte des métiers, de la découverte des formations, de l'étude des entreprises. L'élève doit être capable de compléter ces fiches qui questionnent sa compréhension de la description d'un métier. La COP se charge des activités sur l'identité numérique, ou plutôt, sur la connaissance de soi. Trois activités sont réalisées, un autoportrait, une réflexion sur ses traits personnels, une réflexion sur ses valeurs. Le document de la première séance est repris en classe à la fin du projet et réajusté en fonction des connaissances nouvelles des élèves, l'activité pédagogique est conduite par le binôme enseignant-COP. Ces productions pourraient alimenter une forme de portfolio numérique s'il en existait un, mais en fait, les environnements numériques ne sont pas utilisés pour ces activités.

\subsubsection{Activités constructives}

\subsubsection{Construction du système de ressources}

Des environnements numériques sont utilisés dans la classe de collège, mais uniquement pour ce qui concerne l'orientation ; il s'agit d'une part des environnements d'information développés par l'ONISEP, notamment les ressources sur les métiers et les formations, d'autre part des suites bureautiques comme support des activités.

Le diaporama Exemples d'activités en classe de $5^{\circ}$ qui a été exposé par le binôme enseignant de technologie-COP à la deuxième réunion de recherche pour présenter la déclinaison du projet INO dans la classe de cinquième en collège fournit des données sur le projet, tel que le conçoit le binôme. D'une manière générale, le projet sur l'orientation est articulé avec la maîtrise des compétences informatique et Internet des élèves, il s'agit de "Savoir rechercher et sélectionner des informations" :

- en utilisant les technologies de l'information et de la communication (site meformer.org; guides pour l'orientation GPO-collège ; étude des formations de la sixième à la seconde ; réalisation d'un dossier présentant un métier) ;

- en utilisant des outils pédagogiques ludiques (quiz J'explore les métiers);

- à partir d'activités menées dans la discipline technologie (découverte des métiers exercés dans une entreprise de montage et d'emballage d'un objet).

La dernière activité prévue est un diaporama réalisé individuellement par chaque élève pour présenter son projet d'orientation en lien avec les activités réalisées auparavant sur la connaissance de soi, un secteur 
d'activité avec des métiers qui intéressent l'élève, un choix de parcours de formation. Cette activité n'a pas pu être réalisée, mais l'équipe de recherche a pu visionner des exemples de diaporamas réalisés par des élèves que le professeur avait encadrés au cours de l'année précédente dans le cadre de la préparation "d'une progression sur plusieurs années en cinquième / quatrième / troisième à partir des compétences que l'on doit développer dans le PDMF et au sein de notre programme... en technologie" (extrait du premier entretien).

\subsubsection{Construction d'une démarche réflexive sur soi et e-portfolio}

Dans le cadre de l'orientation, deux documents amènent l'élève à réfléchir sur son parcours scolaire. Dans le premier, l'élève travaille, avec Excel, à une présentation de l'évolution de ses notes ; dans le second, il s'interroge sur son niveau scolaire, les appréciations qu'il reçoit, ses sentiments de réussite et d'échec, ses goûts.

Les activités réalisées sur la connaissance de soi sont toutes de type réflexif ; il s'agit par exemple d'amener l'élève à faire le point sur des valeurs qui lui paraissent importantes, puis à réfléchir à des métiers où ces valeurs pourraient être mises à profit. En revanche, la dimension "identité numérique" n'est pas présente et elle apparaît clairement écartée dans les discours.

\subsubsection{Construction de relations à autrui et e-portfolio}

Autour de la question de l'orientation, les élèves de cinquième travaillent en classe entière ou seuls. Une seule activité mentionne une organisation par groupe des élèves "Par binôme les élèves doivent décrire un métier à partir de leurs connaissances et présenter leur travail oralement." Pour soutenir la progression et conserver la collection des travaux, l'environnement numérique utilisé est le Passeport orientation formation développé par l'ONISEP (Webclasseur). Selon ce qu'en dit l'enseignant, ces environnements ne facilitent pas les échanges "Le Webclasseur est là / on échange / on part toujours de l'espace classe / alors le support / l'élève se l'approprie mais // entre élèves / finalement / il /// permet pas forcément les échanges / si ce n'est dans les mises en commun qui se font dans le cadre de la classe" (entretien de fin de projet).

Autour de la connaissance de soi, les élèves réalisent une activité qui articule la réflexion sur soi et les échanges entre élèves ; dans une liste, chaque élève sélectionne les traits de caractère qu'il s'attribue et fait la même chose avec un camarade de sa classe, puis il les met en parallèle et en déduit ceux qui lui correspondent le plus. Les environnements numériques ne sont pas utilisés pour ces activités.

\subsubsection{Gestion des dispositions à apprendre}

\subsubsection{Sur le plan des artefacts}

La recherche d'informations sur les métiers et les formations s'effectue dans un environnement développé par l'ONISEP ; il s'agit d'un environnement fermé, dédié à cette thématique. Le binôme qui intervient en collège choisit de ne pas aborder la question de l'identité numérique en disant qu'elle ne peut pas être comprise par des élèves de $5^{\circ}$; les activités réflexives sur soi concernent la "connaissance de soi" et se déroulent sous une forme papier-crayon. Les discours laissent entendre que les élèves ont apprécié les activités qui leur ont été proposées, qu'ils n'ont pas rencontré de difficultés avec les outils numériques utilisés (suites bureautiques et environnement de ressources de l'ONISEP), que tous ont réalisé ce qui leur était demandé. Les technologies utilisées semblent acceptables pour les élèves, mais il n'y a pas de mise en place d'un environnement qui s'apparenterait à un e-portfolio. L'enseignant a déposé une fiche d'évaluation de l'environnement, il s'agit d'un questionnaire intitulé Le Webclasseur aide-t-il à s'orienter? qui incite les élèves à répondre de manière anonyme à des questions sur l'utilisabilité de l'environnement, mais aussi sur ce qu'il apporte au projet d'orientation de l'élève. Ce questionnaire n'a cependant pas été utilisé pendant le projet.

\subsubsection{Sur le plan cognitif et affectif}

Le projet a été construit par le binôme, mais essentiellement par l'enseignant et il a été présenté aux élèves. Aucun document et aucun discours ne laissent entendre qu'il y ait eu négociation du projet entre l'enseignant, la COP et les élèves. Le projet général en classe de cinquième présente plusieurs activités dont les objectifs définis sont de motiver les élèves, un temps est notamment consacré à la présentation du 
projet INO devant les élèves en amont du lancement du projet. Les activités sur les outils numériques et sur cet environnement ne semblent pas poser de problèmes spécifiques à des élèves de $5^{\circ}$. Si les élèves sont régulièrement mis en activité individuelle sur fiche, il n'y a pas d'individualisation des parcours d'apprentissages et de développement car les parcours pédagogiques sont tous identiques. Le terme autonomie apparaît à plusieurs reprises dans le discours de l'enseignant, mais lorsqu'il est employé, ce terme concerne toujours des activités que les élèves réalisent seuls par opposition aux activités qu'ils réalisent en classe entière (données issues du tableau Progression pédagogique).

\subsubsection{Analyse du projet INO en classe de $5^{\circ}$}

D'une manière générale, l'enseignant de technologie est très à l'aise avec les activités sur l'orientation en lien avec les outils et ressources numériques développés par l'ONISEP et avec les activités qui permettent de construire les compétences relatives à la maitrise des techniques usuelles de l'information et de la communication. La recherche d'informations apparaît à plusieurs reprises dans le scénario de cinquième et elle revient aussi à d'autres moments de la programmation sur tout le cursus collège. Cette activité qui se déroule plusieurs fois, dans divers contextes, est favorable à la construction d'une instrumentation autour de la recherche d'informations dans des environnements numériques. En revanche, l'absence de dimension réflexive et collaborative sur le numérique est moins propice à la construction d'instruments. Les données analysées ne permettent pas de dire qu'il y a eu individualisation des parcours pédagogiques.

Les activités autour de la construction d'une démarche réflexive sur soi et de la construction de relations sont plus favorables à la construction d'un parcours pédagogique propre à chaque élève. Ces activités sont centrées sur la connaissance de son parcours ou de soi par l'élève et les activités se déroulent sous la forme papier-crayon ; cette individualisation n'est pas articulée avec la dimension numérique du projet. On ne parle d'ailleurs pas d'identité numérique. La réflexion sur le comportement à avoir avec le numérique n'est pas développée.

Le binôme a choisi de travailler avec une séparation nette des thématiques entre les deux intervenants, le professeur de technologie met en œuvre des activités sur l'orientation et utilise le numérique ; la COP met en œuvre des activités sur la "connaissance de soi" sans avoir accès à une salle équipée. L'individualisation des parcours est une préoccupation de la COP, mais ses activités sont déconnectées du numérique. Le professeur de technologie gère la dimension numérique, mais aucune donnée recueillie, de ses discours ou de ses productions, ne permet d'affirmer qu'il a une préoccupation pour l'individualisation des parcours des élèves.

\subsection{Le projet INO dans la classe de 2 nde en anglais}

Si les résultats présentés ici concernent la classe de seconde qui participe au projet avec le professeur d'anglais, il faut toutefois relever que les enseignantes de lycée, bien qu'enseignant des disciplines différentes (anglais et lettres modernes) et intervenant dans des classes différentes, construisent le projet ensemble. Toutes deux commencent par mettre en œuvre les activités sur la connaissance de soi et sur l'identité numérique qu'elles disent plus proches de leurs pratiques disciplinaires. Le cadre pédagogique général articule les thèmes de l'orientation et de l'identité numérique.

Comme pour la technologie en collège, dans la discipline anglais, le projet INO s'intègre au travail réalisé "Avant de poser le cadre pédagogique, il a fallu... Définir le cadre institutionnel (européen, national, régional et au sein de l'établissement); Cerner les objectifs du projet et les rendre pertinents dans la matière" (extrait du diaporama présentant le projet à la seconde réunion en présentiel du groupe). En revanche, cette articulation repose sur un travail préparatoire spécifique de l'enseignante.

Le projet s'intitule I act, I fly, "Un acronyme porteur de sens qui soutient une démarche pédagogique et un apprentissage de la langue dans un cadre technologique". Le projet de la classe articule les dimensions réflexion sur soi et projet de l'élève et leur transfert dans le monde numérique, avec un but final d'autonomie.

Le projet de l'enseignante définit les apprentissages des élèves qui sont visés en termes de compétences :

- "TICE :

- développement d'un mini site personnel par chaque élève, communication et interaction avec gmail 
- Compétences langagières :

- passer de A2 à B1 au fil des étapes du projet

- Compétences transversales : autonomie, sens de l'initiative, vivre en société

- Savoir s'adapter et innover

- Savoir s'exprimer à partir de supports variés

- Apprendre pour agir et agir pour apprendre

- Apprendre à choisir mon orientation en tant que jeune citoyen du monde" (ibidem).

Certaines compétences visées sont directement liées au projet, d'autres sont liées à la discipline dans laquelle le projet s'actualise, enfin, une compétence transversale mérite une mention particulière "Apprendre pour agir et agir pour apprendre" car elle relève des visées du projet de l'enseignant qui est de soutenir l'apprentissage, au-delà des visées strictes du projet. L'enseignante écrit sur une autre diapositive (ibidem) : "Le travail repose complètement sur des concepts et une démarche fortement inspirés par l'approche orientante suggérée par Danielle Ferré [(Ferré, 2005)] et dont la pierre angulaire est la construction de l'identité et l'accompagnement des élèves au sein d'une dynamique de projet dans le cadre scolaire".

A propos de l'identité numérique, elle programme la construction de portraits, un travail porte sur "comment établir un profil personnel positif", un autre sur les personnages auxquels on s'identifie avec une réflexion sur les raisons qui poussent à ces choix, des activités de création "Travail sur le thème "I dream »". Les productions des élèves sont déposées sur leurs blogs individuels.

Les activités sur l'orientation viennent après les activités sur la connaissance de soi et sur l'identité numérique. Autour de l'orientation, les activités sont un choix de métiers "Réfléchir à 2 métiers et ouvrir deux profils virtuels" associé à une réflexion sur l'orientation vers ces métiers, une réflexion sur la mobilité internationale, mais aussi des activités plus pratiques comme la rédaction d'une courte lettre de motivation ou d'un "mini $C V$ ". Ces productions sont également déposées dans les blogs.

\subsubsection{Activités constructives}

\subsubsection{Construction du système de ressources}

Les environnements numériques utilisés dans le projet INO dans la classe de seconde avec le professeur d'anglais sont d'une part le blog ("Ouverture d'un mini site par élève ; Transfert de contenu ; Relier les mini sites"), d'autre part le Web pour trouver des informations sur les métiers, l'établissement ne dispose pas de la connexion aux ressources développées par l'ONISEP. L'enseignante met également en place une formation sur l'utilisation du présenteur.

L'enseignante ouvre un blog dont la première page présente le projet INO pour la classe concernée. Elle y présente notamment un diagnostic initial dans lequel elle écrit que l'orientation traditionnelle ne prend pas en compte "l'évolution très rapide des technologies largement utilisées par les élèves, la société civile et dans le monde du travail, d'où la nécessité de rechercher des façons de permettre aux jeunes utilisateurs du web 2.0 de mieux maîtriser leur identité, leur identité numérique et de la faire évoluer pour qu'elle leur ouvre les portes de leur avenir en tant que citoyens responsables." (première page du site de classe). Cette enseignante relie ainsi explicitement l'utilisation des technologies numériques à l'individualisation des parcours pédagogiques des élèves.

\subsubsection{Construction d'une démarche réflexive sur soi et e-portfolio}

La réflexion sur soi est présente dans les activités sur l'identité, sur l'identité numérique et sur l'orientation "Mieux me connaître (identité) et apprendre à transférer mes compétences pour mieux réussir à l'école et après l'école" (extrait du diaporama présentant le projet à la seconde réunion en présentiel du groupe) ; les élèves doivent réfléchir sur eux-mêmes, sur la manière dont ils envisagent leur contribution à la société, sur leur parcours en rédigeant un curriculum vitae succinct qu'ils déposent dans leur blog. Dans les activités centrées sur la réflexion sur soi, les dimensions pragmatique "I can" et 
existentielles "I dream" de l'identité (Esparbes-Pistre et Tap.2000) sont présentes.

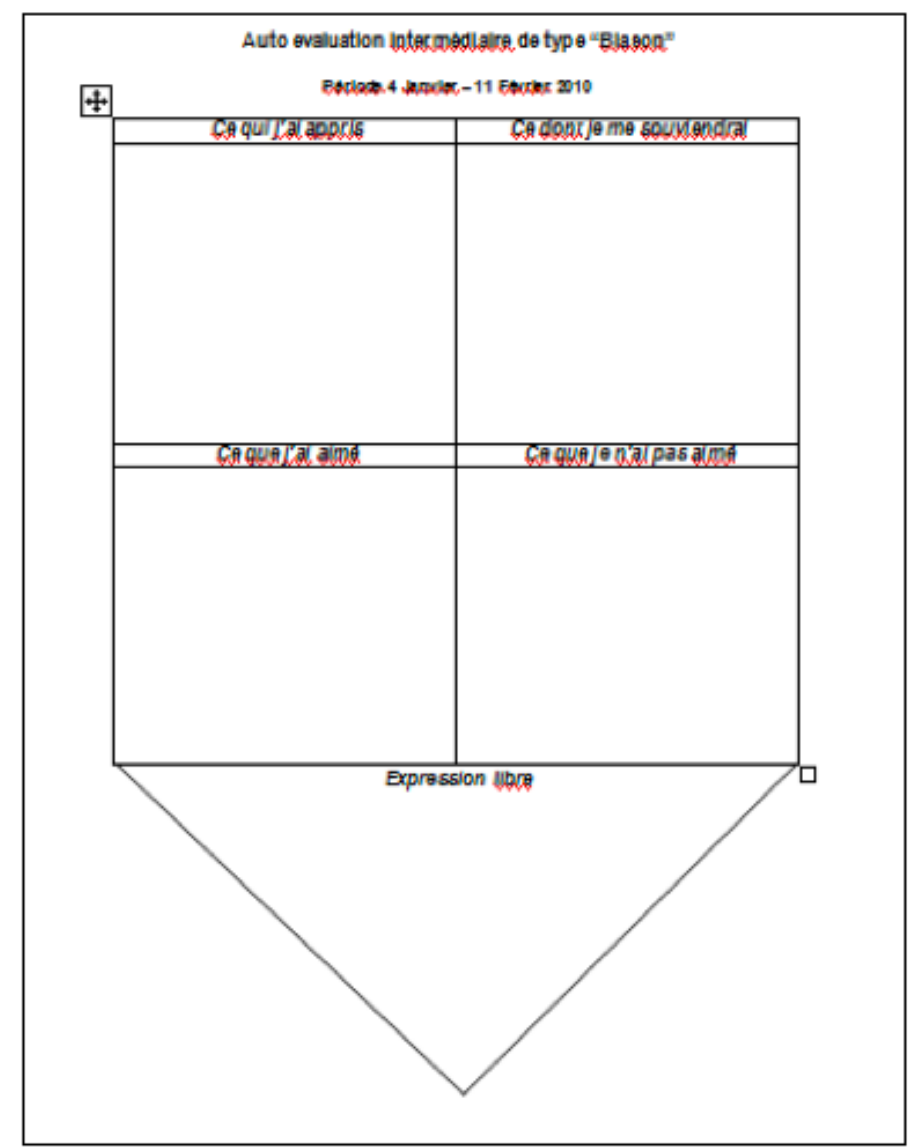

Figure 4 • Activités réflexives en classe de $2^{\text {nde }}$ en anglais

Les activités réflexives sont également présentes lors des auto-évaluations, par exemple, au milieu du projet, l'élève est invité à faire le point et à écrire ce qu'il a appris, ce dont il pense se souvenir, ce qu'il a aimé et ce qu'il n'a pas aimé pendant la première phase du projet. Un espace est également dédié à l'expression libre. L'individualisation du parcours s'actualise dans une activité d'auto-évaluation.

\subsubsection{Construction de relations à autrui et e-portfolio}

Par le projet, il est attendu que l'élève s'ouvre aux autres élèves de la classe et au monde extérieur avec l'objectif de "vivre en société" et d'apprendre à choisir son orientation "en tant que jeune citoyen du monde".

Les activités collaboratives sont très présentes dans le projet en classe de seconde "Découvrir mes opportunités de carrière au contact des autres (collaboration) et apprendre à construire mes choix" (ibidem). Elles prennent la forme de travail par groupes d'élèves, d'échanges entre élèves, etc., et visent la collaboration "Collaboration avec personnes et documents ressources pour apprendre à m'orienter vers ces carrières potentielles" et la compréhension de liens entre ce qui se fait en milieu scolaire et ce qui existe dans le monde "Comprendre le lien entre mon apprentissage scolaire et le monde extérieur". Pendant les activités d'auto-évaluation, il est demandé aux élèves de noter leur degré d'implication (de 1 à 5) dans le travail collectif “Le projet m'a permis... d'aider les autres sans qu'on me le demande", mais aussi de positionner (de 1 à 5) l'effet du projet sur le travail de groupe "Le travail de groupe et l'interactivité sont facilités par ce type de projet".

Niveau outils, le blog et la messagerie soutiennent les interactions des élèves "communication et interaction avec gmail". Ils permettent aussi des interactions à distance et hors temps scolaire avec l'enseignante.

\subsubsection{Gestion des dispositions à apprendre}




\subsubsection{Sur le plan des artefacts}

Dans le projet, chaque élève réalise son blog, même si les activités qui y sont réalisées sont en lien avec les activités scolaires et présentent de ce fait une certaine similitude. Pour les blogs, il y a un "Modèle de base identique à personnaliser au gré $d u$ projet"; il y a donc individualisation des parcours. L'enseignante s'intéresse à la créativité et elle porte une attention au projet de chacun ' $j$ 'ai pris les élèves les uns derrière les autres / $j$ 'ai regardé leur site Web et puis j'en ai discuté avec eux et on a mis des notes d'un commun accord /// c'était un petit peu travailler avec les élèves sur comprendre comment eux ils ressentaient //I et /// comment moi je comprenais la façon dont ils ont travaillé". Pour voir s'il était possible d'aller plus loin dans l'individualisation avec les blogs, il aurait été nécessaire que le projet soit reconduit plusieurs années avec les mêmes élèves.

L'enseignante d'anglais anticipe l'utilisation des outils numériques par les élèves "Préparer et sélectionner les outils de communication que... les élèves vont utiliser... [ils] doivent se sentir à l'aise et pouvoir communiquer facilement". L'enseignante crée aussi son propre blog pour se présenter et le met à disposition de ses élèves pour les aider à comprendre ses attentes.

\subsubsection{Sur le plan cognitif et affectif}

C'est l'enseignante qui définit les objectifs visés et les temps d'apprentissage, la production attendue et le délai, qui décide de l'environnement qui va accueillir les blogs, mais les élèves bénéficient d'une liberté pour concevoir leur projet. Le projet de l'enseignante accorde une place importance à la réflexion sur soi et au travail collectif des élèves. Il y a donc individualisation des parcours d'apprentissage et de développement. Les évaluations participent aussi de l'individualisation; elles sont transmises aux élèves dès le lancement du projet pour les inciter à prendre en charge leurs apprentissages, puis pendant le projet, l'évaluation jalon (figure 5) les incite à revenir sur leurs parcours.

Dans le projet mené en classe de seconde en anglais, la motivation est soutenue tout au long du projet par 1'“acronyme porteur de sens" qui est à la fois un nom pour le projet et un programme d'actions. Tout au long du projet, l'enseignante reste disponible à distance et hors temps scolaire, car, du fait que les sites sont dans une langue étrangère, les élèves attendent des feed-back "ils avancent... plus lentement et en plus après ils n'osent pas mettre en ligne donc ils m'envoient des mails / il faut que je regarde / ils me demandent de corriger / alors moi j'avance à petits pas mais c'est parce qu'il y a la barrière de la langue étrangère". La gestion des espaces et des temps pédagogiques participe de l'individualisation. L'enseignante rassure également les élèves qui " $n$ 'ont pas // complètement compris cette façon d'apprendre / intégrée à une démarche"; le développement passe par une mise en évidence de l'articulation du projet avec les apprentissages liés au programme "de temps en temps il fallait que je fasse des pauses en leur disant voilà où on en est" (entretien de fin de projet). Les apprentissages de la discipline sont liées à la visée élargie du projet.

\subsubsection{Analyse du projet INO en classe de 2 nde en anglais}

L'enseignante d'anglais est à l'aise avec les outils numériques et elle fait preuve d'une grande réflexion sur l'apprentissage. L'importance qu'elle accorde à la réflexion sur soi et au travail collectif va dans le sens d'une perception d'un élève capable de créer, de faire des choix, de réfléchir en autonomie, d'échanger avec ses pairs, activités qui participent de l'individualisation des apprentissages.

Le projet s'inscrit dans une perspective développementale dès le départ comme le montre la diapositive finale du diaporama présentant l'individualisation dans son projet à la seconde réunion en présentiel du groupe. La prise en charge par l'élève de son site, une perspective évolutive, une volonté d'aller au-delà de l'immédiateté du temps scolaire, une perspective à long terme apparaissent clairement. Une analyse complète du scénario construit par cette enseignante et des ressources qu'elle a utilisées a montré qu'il s'agit d'un enseignement ancré dans le socioconstructivisme (Loisy et al., 2010). Les élèves sont actifs et leurs activités dans le e-portfolio participent des médiations pragmatiques, épistémiques, réflexives et interpersonnelles.

L'individualisation des apprentissages et du développement est présente dans les discours et dans les activités qu'elle propose. 


\section{Intérêt des blogs dans l'individualisation de parcours d'apprentissage}

L'équipe de recherche a mis en place un dispositif d'accompagnement et un site collaboratif, mais les enseignants n'étaient pas contraints à choisir le même environnement. Cette liberté qui leur est offerte leur permet de choisir librement l'environnement qui servira de base pour les e-portfolios d'élèves, les tâches qu'ils vont réaliser en classe, les objets sur lesquels ils vont se focaliser. De ce fait, les environnements qu'ils choisissent sont nécessairement en adéquation avec leurs activités, leurs choix pédagogiques, leurs connaissances des élèves. Les participants construisent et mettent en œuvre des activités dans le cadre du projet, mais des différences importantes peuvent être observées, au niveau de l'organisation pédagogique dans les classes de cinquième et de seconde, au niveau des ressources numériques, au niveau de la mise en place du e-portfolio.

Au collège, bien que l'enseignant soit très à l'aise avec les outils numériques, le projet qu'il construit n'a pas toutes les caractéristiques attendues et il n'y a pas de mise en place de e-portfolios. Si l'on regarde du côté des situations qui existent dans la classe de cinquième en ce qu'elles contribuent au développement des élèves en permettant une appropriation des outils et le développement d'un instrument, l'appropriation porte sur les suites bureautiques et sur les environnements de ressources (les élèves sont capables, en fin de projet, d'alimenter une présentation de leur projet avec des ressources qu'ils sont allés rechercher dans les environnements de ressources développés par l'ONISEP) et sur la connaissance de l'environnement éducatif en lien avec la question de l'orientation (le questionnaire d'évaluation de connaissances de fin d'année révèle qu'ils connaissent mieux, en fin de projet, les sites de ressources sur les métiers et les personnes susceptibles de les conseiller quant à leur orientation). Le projet soutient de manière volontariste les médiations pragmatiques, mais ne reste centré que sur elles. Les autres médiations sont moins présentes dans les discours et dans les productions de l'enseignant. Il n'est pas possible d'affirmer, suite à l'analyse des documents produits et des discours, qu'il y ait eu individualisation des apprentissages et du développement. La séparation entre les activités de la COP et de l'enseignant ne facilite pas la construction d'un parcours global par l'élève, l'individualisation est peu prise en compte.

Au lycée, l'enseignante fait créer des blogs à ses élèves. Ces blogs ont les caractéristiques attendues des e-portfolios. Les activités conduites concernent l'identité numérique et l'orientation. Les compétences visées couvrent une large palette qui dépasse le projet sur l'identité numérique et l'orientation ; certaines sont directement liées au projet (compétences liées aux technologies numériques ; compétences liées à la construction du projet d'orientation ; compétences transversales), d'autres à la discipline "langue étrangère" (compétences langagières générales "Savoir s'exprimer à partir de supports variés" et disciplinaires "passer de A2 à B1 au fil des étapes du projet"). Enfin, comme il a été souligné, la compétence transversale "Apprendre pour agir et agir pour apprendre" relève des visées du projet de l'enseignant de soutenir l'individualisation des apprentissages et du développement. L'enseignante soutient la construction du parcours global par l'élève, on peut donc dire que l'individualisation est bien prise en compte. L'inscription de cette compétence transversale dans le projet reflète également une posture épistémologique qui est en accord avec le cadre théorique général qui guide la recherche INO. Si l'on regarde du côté des situations qui ont existé dans la classe de seconde en ce qu'elles ont contribué au développement des élèves en permettant une appropriation des outils et le développement d'instrument, on observe que la production du blog est totalement articulée au projet personnel et professionnel des élèves. Par ailleurs, il est attendu que les élèves collaborent entre eux et avec des personnes extérieures. La dimension réflexive est présente autour de l'identité numérique et de l'orientation, mais aussi au niveau des relations interpersonnelles, par exemple lorsque l'élève doit penser à sa contribution citoyenne au monde. Le projet soutient les médiations pragmatiques, les médiations épistémiques, les médiations réflexives et les médiations interpersonnelles. Au niveau de l'étayage, on peut observer que les élèves réalisent des activités adaptées à leur âge et que l'enseignante a le souci de les motiver. Elle n'hésite pas à se lancer dans la réalisation de son propre blog pour en comprendre la dynamique ; cela donne aussi un exemple de blog à ses élèves pour qu'ils se lancent dans la réalisation du leur. Elle montre un souci permanent d'approcher au plus près les exigences du programme que les élèves lui rappellent avec les 
buts qu'elle s'est fixés pour le projet INO. Il y a, tant sur le plan technique que sur le plan de l'accompagnement humain, une volonté de mettre en place une individualisation des apprentissages et du développement. Cette volonté apparaît aussi bien dans les activités qu'elle propose à ses élèves que dans les discours qu'elle tient. Le projet de seconde en anglais vise la construction de ressources "réutilisables dans le cadre de son cursus d'apprentissage". L'individualisation est présente également dans l'attention que l'enseignante porte au projet de chacun de ses élèves.

Par rapport aux visées du projet, les résultats montrent que les productions s'inscrivent dans les trois rapports au monde, on observe des activités constructives en lien avec la construction du système de ressources, de la construction d'une démarche réflexive sur soi, de la construction d'une socialisation. L'accompagnement vise l'instrumentation au niveau des outils, mais également les plans cognitifs et affectifs. Les projets de classe visent, malgré leurs différences, l'individualisation des apprentissages avec une visée d'individualisation du développement.

Le projet INO introduit de la nouveauté, des questions inédites pour les enseignants, et de la complexité dans la classe. La nouveauté vient du fait que la question de l'identité numérique n'a jamais été abordée par les praticiens participant au projet et que l'orientation ne l'était pas auparavant en lycée. La complexité vient de la demande de la recherche de penser l'articulation de l'identité numérique et de l'orientation et de mettre en auvre un environnement numérique permettant de soutenir l'individualisation des apprentissages et du développement, de type portfolio numérique, avec introduction d'une démarche réflexive et collaborative. Une autre originalité de ce projet réside dans une approche qui privilégie la psychologie du développement pour soutenir la construction d'ingénieries pédagogiques articulant une entrée très engageante pour les élèves (leur projet d'orientation, la construction de leur identité, la question de la visibilité de soi...) avec l'utilisation d'environnements numériques. Continuer à développer ce modèle pourrait ouvrir des pistes pour les ingénieries pédagogiques s'appuyant sur les technologies numériques dans le système éducatif où la question de la construction et de l'évaluation des compétences est devenue prégnante.

(1) La recherche INO est conduite dans le cadre d'une convention entre l'IFE (Institut Français de l'Education, ex-INRP) et la Direction générale de l'enseignement scolaire (DGESCO) du Ministère de l'éducation nationale finançant l'étude et l'accompagnement du projet Pairform@nce, (http://national.pairformance.education.fr/) programme national de formation continue des enseignants du premier et du second degré. Le consortium de recherche coordonné par l'équipe EducTice de l'IFE a produit plusieurs rapports de recherche (Gueudet et al., 2008) ; (Soury-Lavergne et al., 2009) ; (SouryLavergne et al., 2011). Le dispositif Pairform@nce permet de concevoir des parcours de formation qui sont ensuite à disposition de formateurs afin qu'ils puissent mettre en œuvre des formations s'appuyant sur ces parcours.

(2) MEN-DGESCO, Circulaire n 2009-068 du 20-5-2009

(3) Le socle commun de connaissances et de compétences (Décret $n^{\circ} 2006-830$ du 11 juillet 2006) est le référentiel qui définit les sept compétences que tout élève doit valider en vue de l'obtention du diplôme national du brevet (DNB).

(4) Parcours de Découverte des Métiers et des Formations (PDMF) et Passeport Orientation Formation (Webclasseur).

(5) Les points de suspension entre parenthèses carrées indiquent qu'une partie du discours a été coupée. Les marques / et // renvoient à des pauses naturelles dans les discours des répondants.

\section{REMERCIEMENTS}

Je tiens à remercier Stéphanie Mailles-Viard Metz et Pierre Bénech qui ont collaboré à tous les niveaux de la recherche, ainsi que les enseignants et la conseillère d'orientation-psychologue des deux établissements de l'académie de Montpellier qui ont participé à la recherche, enfin l'ONISEP LanguedocRoussillon et le SAIO de l'Académie de Montpellier qui ont soutenu le projet. 


\section{BIBLIOGRAPHIE}

ALLAL, L. (1999). Impliquer l'apprenant dans le processus d'évaluation : promesses et pièges de l'autoévaluation. In C. Depover et B. Noël (Eds.), L'évaluation des compétences et des processus cognitifs, p. 35-56. Bruxelles : De Boeck.

BEGUIN, P., RABARDEL, P. (2000). Designing for instrument-mediated activity. Scandinavian Journal of Information Systems, Vol. 12, p. 173-191.

BRUNER, J.S. (1983). Le développement de l'enfant. Savoir faire, savoir dire. Paris : PUF.

BRUNER, J.S. (1987). Comment les enfants apprennent à parler. Paris : Retz

CARDON, D. (2008). Réseaux sociaux de l'Internet. Réseaux, Vol. 6/2008, n 152, p.7-17.

DESGAGNE, S. (1997). Le concept de recherche collaborative : l'idée d'un rapprochement entre chercheurs universitaires et praticiens. Revue des Sciences de l'Education, Vol. 23 n², p. 371-393.

ESPARBES-PISTRE, S., TAP, P. (2000). Identité, projet et adaptation selon les âges de la vie. Carriérologie : revue francophone internationale. Vol. $8 \mathrm{n}^{\circ} 1-2$.

FERRE, D. Pour une approche orientante de l'école française. Paris : Editions Qui plus est.

GEORGE, F. (2009). Approche statistique de trois composantes de l'identité numérique dans Facebook. In F. Millerand, S. Proulx, J. Rueff (dir.), Web social. Mutation de la communication p.187-203. Québec : Presses universitaires du Québec.

GUEUDET, G., SOURY-LAVERGNE, S., TROUCHE, L. (dir.). (2008). Vers des assistants méthodologiques pour les professeurs. Rapport de recherche. Lyon : INRP.

GUICHARD, J, HUTEAU, M. (2006). Psychologie de l'orientation (2éd.). Paris : Dunod.

JORRO, A. (2009). La construction de l'éthos professionnel en formation alternée. Travail et apprentissage, Vol. 3, p.13-25.

LAYEC, J. (2006). Auto-orientation tout au long de la vie : le portfolio réflexif. Paris, France : L'Harmattan.

LINARD, M. (2002). Conception de dispositifs et changement de paradigme en formation. Education Permanente, $\mathrm{n}^{\circ} 152$, Regards multiples sur les nouveaux dispositifs de formation, p. 143-155.

LOISY, C., MAILLES-VIARD METZ S., BENECH, P. (2010). Scénarios pour l'identité numérique et la construction de l'orientation. In M. Sidir, E. Bruillard et G.-L. Baron, Acteurs et Objets Communicants. Vers une éducation orientée objets ? p. 224-236. Lyon : INRP.

LOISY, C., BRETON, H., MAILLES-VIARD METZ S. (2011). E-portfolio et orientation : quel(s) accompagnement(s) pour la construction du parcours professionnel ? Revue Internationale des Technologies de l'Enseignement Supérieur, 2011, Vol. $8 \mathrm{n}^{\circ} 1-2$, éditeurs G. Gueudet, G. Lameul et L. Trouche. Disponible en ligne : http://www.ritpu.org/spip.php?rubrique63\&lang=fr

MERZEAU, L. (2010). La présence plutôt que l'identité. Documentaliste - sciences de l'information, Vol. 47 nº 1, p. 32-33.

PASTRE, P. (2005). La conception de situations didactiques à la lumière de la théorie de la conceptualisation dans l'action. In $\mathrm{P}$. Rabardel et P. Pastré (Eds.), Modèles du sujet pour la conception. Dialectiques activités développement, p. 73-108. Toulouse : Octarès Editions.

PELLETIER, D., DUMORA, B. (1984). Fondements et postulats pour une conception éducative de l'orientation. In D. Pelletier et R. Bujold (dir.) Pour une approche éducative en orientation p. 27-37. Chicoutimi (Québec) : Gaëtan Morin.

PIAGET, J. (1937). La construction du réel chez l'enfant. Neuchâtel ; Paris : Delachaux et Niestlé

QUERE, L. (2000). Singularité et intelligibilité de l'action. In J.-M. Barbier, Y. Clot, F. Dubet, O. Galatanu, M. Legrand, J. Leplat, M. Maillebouis, J.L. Petit, L. Quéré, J. Theureau, L. Thévenot, P. Vermersch, L'analyse de la singularité de l'action p. 147-170. Paris : Presses Universitaires de France.

RABARDEL, P. (1995). Les hommes et les technologies : une approche cognitive des instruments contemporains. Paris : Armand Colin.

RABARDEL, P. (2005/2009). Instrument subjectif et développement du pouvoir d'agir. In P. Rabardel et P. Pastré (Eds.), Modèles du sujet pour la conception. Dialectiques activités développement. Toulouse : Octarès Editions.

RABARDEL, P. et SAMURÇAY, R. (2003). Artifact mediated Learning. In Y. Engeström et M. Hasu (Eds.), New Challenges to research on Learning. Hillsdale (NJ): Lawrence Erlbaum.

SCHON, D. (1983). Reflective Practitioner: How professionals think in action. London: Temple Smith.

SOURY-LAVERGNE, S., GUEUDET, G., TROUCHE, L. (Eds.). (2009). Parcours de formation en ligne, étude de processus 
d'appropriation. Rapport de recherche. Lyon. INRP.

SOURY-LAVERGNE, S., GUEUDET, G., LOISY, C., TROUCHE, L. (Eds.). (2011). Parcours de formation, de formateurs et de stagiaires : suivi et analyse. Rapport de recherche. Lyon. INRP.

TAP, P. (1979). Relations interpersonnelles et genèse de l'identité. Homo, Vol. 18, p. 7-43.

TRICOT, A. et TRICOT, M. (2000). Un cadre formel pour interpréter les liens entre utilisabilité et utilité des systèmes d'information. Actes du Colloque Ergo-IHM 2000 p. 195-202, Biarritz, 3-6 octobre.

VARGA, R., CARON, P.-A. (2009). Persistance des connaissances construites et perméabilité des réseaux sociaux : construire un lien entre les plateformes de formation et les environnements privés. Actes du colloque EPAL 2009 (Echanger pour apprendre en ligne : conception, instrumentation, interactions, multimodalité), université Stendhal - Grenoble 3, 5-7 juin 2009.

VERMERSCH, P. (2000). Approche du singulier. In J.-M. Barbier, Y. Clot, F. Dubet, O. Galatanu, M. Legrand, J. Leplat, M. Maillebouis, J.L. Petit, L. Quéré, J. Theureau, L. Thévenot, P. Vermersch, L'analyse de la singularité de l'action, p. 239-256. Paris : Presses Universitaires de France.

VYGOTSKI, L. (1934/1985). Pensée et langage. Paris : Editions sociales.

\section{A propos de l'auteur}

Catherine LOISY est maître de conférences en psychologie. Elle est actuellement détachée dans l'équipe EducTice-S2HEP de l'Institut français de l'éducation (IFÉ), ENS de Lyon. Ses domaines de recherche sont la psychologie cognitive et la psychologie du développement. Ses thématiques actuelles sont centrées sur le développement professionnel des enseignants, notamment sur les portfolios et les compétences numériques.

Adresse : EducTice / Institut Français d'Éducation (IFÉ), École Normale Supérieure de Lyon, 15 parvis René-Descartes, BP 7000, 69342 Lyon cedex 07

Courriel : $\underline{\text { catherine.loisy@ens-lyon.fr }}$

Toile : $\underline{\text { http://eductice.inrp.fr/EducTice/equipe/membres/permanents/catherine-loisy }}$

Référence de l'article :

Catherine LOISY, Individualisation de parcours d'apprentissage : potentiel de blogs, Revue STICEF, Volume 19, 2012, ISSN : 1764-7223, mis en ligne le 21/11/2012, http://sticef.org

(C) Revue Sciences et Technologies de 1'Information et de la Communication pour l'Éducation et la Formation, 2012 